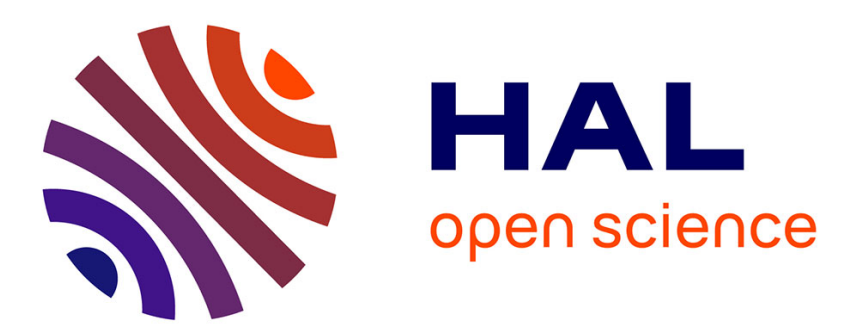

\title{
A numerical study of the size of the homoclinic tangle of hyperbolic tori and its correlation with Arnold diffusion in Hamiltonian systems
}

\author{
Elena Lega, Massimiliano Guzzo, Claude Froeschlé
}

\section{To cite this version:}

Elena Lega, Massimiliano Guzzo, Claude Froeschlé. A numerical study of the size of the homoclinic tangle of hyperbolic tori and its correlation with Arnold diffusion in Hamiltonian systems. Celestial Mechanics and Dynamical Astronomy, 2010, 107 (1-2), pp.129-144. 10.1007/s10569-010-9264-8 . hal00552506

\section{HAL Id: hal-00552506 https://hal.science/hal-00552506}

Submitted on 6 Jan 2011

HAL is a multi-disciplinary open access archive for the deposit and dissemination of scientific research documents, whether they are published or not. The documents may come from teaching and research institutions in France or abroad, or from public or private research centers.
L'archive ouverte pluridisciplinaire HAL, est destinée au dépôt et à la diffusion de documents scientifiques de niveau recherche, publiés ou non, émanant des établissements d'enseignement et de recherche français ou étrangers, des laboratoires publics ou privés. 


\title{
A numerical study of the size of the homoclinic tangle of hyperbolic tori and its correlation with Arnold diffusion in Hamiltonian systems
}

\author{
Elena Lega · Massimiliano Guzzo • Claude Froeschlé
}

Received: 12 November 2009 / Revised: 24 February 2010 / Accepted: 26 February 2010 /

Published online: 27 March 2010

(C) Springer Science+Business Media B.V. 2010

\begin{abstract}
Using a three degrees of freedom quasi-integrable Hamiltonian as a model problem, we numerically compute the unstable manifolds of the hyperbolic manifolds of the phase space related to single resonances. We measure an exponential dependence of the splitting of these manifolds through many orders of magnitude of the perturbing parameter. This is an indirect numerical verification of the exponential decay of the normal form, as predicted by the Nekhoroshev theorem. We also detect different transitions in the topology of these manifolds related to the local rational approximations of the frequencies. The variation of the size of the homoclinic tangle as well as the topological transitions turn out to be correlated to the speed of Arnold diffusion.
\end{abstract}

Keywords Homoclinic tangle · Nekhoroshev theorem · Hyperbolic manifolds · Diffusion · Chaotic hyperbolic section

\section{Introduction}

The problem of long term stability in Hamiltonian systems is strongly related to the celebrate KAM and Nekhoroshev theorems which leave the possibility for a drift of orbits on a special subset of the phase space, the so called Arnold web. Concerning the dynamics in the Solar System we can find examples of slow diffusion through resonances in the outer planetary system (Guzzo 2005, 2006) and among the Trojan asteroids (Robutel and Gabern 2006). Though usual problems in Celestial Mechanics are represented by degenerate Hamiltonian systems, which do not directly satisfy the hypothesis of Nekhoroshev (1977) and KAM theorem, the

E. Lega $(\bowtie) \cdot$ C. Froeschlé

Observatoire de Nice, Université de Nice Sophia Antipolis, CNRS UMR 6202,

Bv. de l'Observatoire, B.P. 4229, 06304 Nice Cedex 4, France

e-mail: elena@obs-nice.fr

M. Guzzo

Dipartimento di Matematica Puraed Applicata, Università degli Studi di Padova, via Trieste 63, 35121 Padova, Italy 
degeneracy can be removed in some interesting domains of the phase space by perturbation techniques (Arnold 1963; Robutel 1995; Locatelli and Giorgilli 2005, 2007; Celletti and Chierchia 2006, 2007; Guzzo and Morbidelli 1997; Morbidelli and Giorgilli 1997; Guzzo 1999). Then, the effective applicability of KAM and Nekhoroshev theorems can be checked with computer assisted techniques, as it was done in Locatelli and Giorgilli (2005), Locatelli and Giorgilli (2007), Celletti and Chierchia (2006), Celletti and Chierchia (2007) for the KAM theorem, or with numerical techniques, as it was done in Guzzo et al. (2002), Pavlovic and Guzzo (2008) for real asteroids of the main belt. The general mechanism which produce chaos and diffusion in the Arnold web is still the subject of numerical and theoretical studies (Chierchia and Gallavotti 1998; Bessi 1996, 1997; Bessi et al. 2001; Berti and Bolle 2002; Berti et al. 2003; Lega et al. 2003; Guzzo et al. 2005; Froeschlé et al. 2005; Todorovic̀ et al. 2008). In a previous paper (Lega et al. 2009) we have studied one of the most popular mechanisms for the production of chaos and diffusion in quasi-integrable systems, which is related to the so called homoclinic tangle of the hyperbolic orbits in the single resonances. More precisely we have provided a measure of the splitting of the normally hyperbolic invariant manifolds of a 4 dimensional symplectic mapping in the specific case of validity of the Nekhoroshev theorem. The measure of the splitting was obtained with two different methods: first we measured the size of the lobes of the homoclinic tangle and second, we measured the size of the associated chaotic zone. We numerically found with both methods of analysis that the chaotic zone $S$ decreases approximately exponentially with the inverse of the perturbing parameter $\epsilon$. Moreover, we have fitted the quantity $S$ versus the diffusion coefficient $D$ by the law: $D \propto S^{2.36}$. These results provide first, an indirect numerical verification of the exponential decay of the normal form as predicted by Nekhoroshev theorem, and second, an indication of the correlation among the exponentially small remainder and the diffusion coefficient $D$.

For quasi-integrable Hamiltonian systems with more than 2 degrees of freedom the splitting of separatrices has a crucial interest for the problem of Arnold diffusion (Arnold 1964; Simó and Valls 2001). It is known from the work of Neishtadt (1984) that the splitting of separatrices cannot exceed the size of the remainder of the normal form. Using heuristic arguments and numerical experiments Morbidelli and Giorgilli (1997) have shown that for generic quasi-integrable Hamiltonian systems the amplitude of the splitting, being related to the coefficient of leading secondary resonances, is the same as the size of the remainder of the optimal normal form. An analytical investigation can be found on Efthymiopoulos (2008) concerning the quasi-integrable Hamiltonian introduced in Froeschlé et al. (2000) while, at our knowledge, the problem has not been yet studied numerically on more generic Hamiltonian systems.

In this paper, we compute the size of the chaotic zone related to the remainder of the normal form of single resonances for the Hamiltonian model introduced in Froeschlé et al. (2000) and studied by semi-analytical tools in Efthymiopoulos (2008).

We confirm the results obtained for the mapping concerning the exponential decrease of the size of the chaotic zone and the power law relation between this latter and the diffusion coefficient. We compare our numerical results to the analytical ones for the specific resonance studied in Efthymiopoulos (2008). Moreover, we have recently studied (Guzzo et al. 2009a) the topology of the stable and unstable manifolds of the normally hyperbolic invariant manifolds of the mapping studied in Lega et al. (2009). In the present paper we study the topology of (un)stable manifolds of the Hamiltonian model. We show that for the Hamiltonian case, as well as obtained for the mapping (Lega et al. 2009), the transitions observed in the topology are correlated to the changes in the behaviour of the diffusion coefficient. 
The paper is organized as follows: in Sect. 2 we define our model problem of quasi-integrable system; in Sect. 3 we detect the exponential decreasing of the size of the chaotic zone related to the asymptotic manifolds in the single resonances; in Sect. 4 we study the relation with the diffusion coefficient. We provide in Sect. 5 the study of the topology of asymptotic manifolds and we relate the topological transitions to the diffusion coefficient. Conclusions are provided in Sect. 6 .

\section{A model of quasi-integrable system}

In this paper we refer to the Hamiltonian system introduced in Froeschlé et al. (2000) and studied in Guzzo et al. (2005):

$$
H=\frac{I_{1}^{2}}{2}+\frac{I_{2}^{2}}{2}+I_{3}+\epsilon \frac{1}{\cos \left(\varphi_{1}\right)+\cos \left(\varphi_{2}\right)+\cos \left(\varphi_{3}\right)+4}
$$

where $I_{1}, I_{2}, I_{3} \in \mathbb{R}$ and $\varphi_{1}, \varphi_{2}, \varphi_{3} \in \mathbb{T}$ are canonically conjugate and $\epsilon$ is a small parameter.

The Hamiltonian (1) has the following invariant manifold:

$$
\Lambda=\left\{\left(I_{1}, \varphi_{1}, I_{2}, \varphi_{2}, I_{3}, \varphi_{3}\right): \text { such that }\left(I_{1}, \varphi_{1}\right)=(0, \pi)\right\}
$$

for any value of $\epsilon .^{1}$

The dynamics of the system on the manifold $\Lambda$ is represented by the following Hamiltonian system:

$$
H_{\Lambda}=\frac{I_{2}^{2}}{2}+I_{3}+\epsilon \frac{1}{\cos \left(\varphi_{2}\right)+\cos \left(\varphi_{3}\right)+3}
$$

or equivalently by the second order equation:

$$
\ddot{\varphi}_{2}=-\epsilon \frac{\sin \left(\varphi_{2}\right)}{\left(\cos \left(\varphi_{2}\right)+\cos (t)+3\right)^{2}} .
$$

Therefore, the dynamics restricted to $\Lambda$ is also quasi-integrable, and the KAM theorem applies to (3).

It appears clearly (see Fig. 1) that, for $\epsilon=0.4$, the Poincaré section of (3) has no more invariant KAM tori which bound the possibility of chaotic diffusion as it is in Fig. 1, left for $\epsilon=0.01$. Using Poincaré sections we have checked that for $\epsilon<\epsilon_{c} \simeq 0.4 \mathrm{KAM}$ tori bound the possibility of chaotic diffusion.

In Sects. 3 and 4 we study the stable and unstable manifolds of a selected two-dimensional invariant torus of $\Lambda$, precisely the one with frequencies:

$$
\bar{\omega}=\left(\bar{\omega}_{2}, \bar{\omega}_{3}\right)=\left(\frac{\sqrt{5}-1}{2}, 1\right),
$$

Through frequency analysis we have found that this torus exists at least up to $\epsilon=0.1$.

Let us remark that our numerical experiments are made in the regime of validity of the Nekhoroshev theorem. We have checked through a method developed in Froeschlé et al. (2000), that the system is in the Nekhoroshev regime up to $\epsilon \simeq 0.03$, which turns out to be one order of magnitude lower than the threshold of transition to diffusion on $\Lambda$.

1 The manifold $\Lambda_{2}=\left\{\left(I_{1}, \varphi_{1}, I_{2}, \varphi_{2}, I_{3}, \varphi_{3}\right):\right.$ such that $\left.\left(I_{2}, \varphi_{2}\right)=(0, \pi)\right\}$ has the same characteristic as $\Lambda$. Therefore the numerical experiences all over the paper could be repeated for $\Lambda_{2}$. 

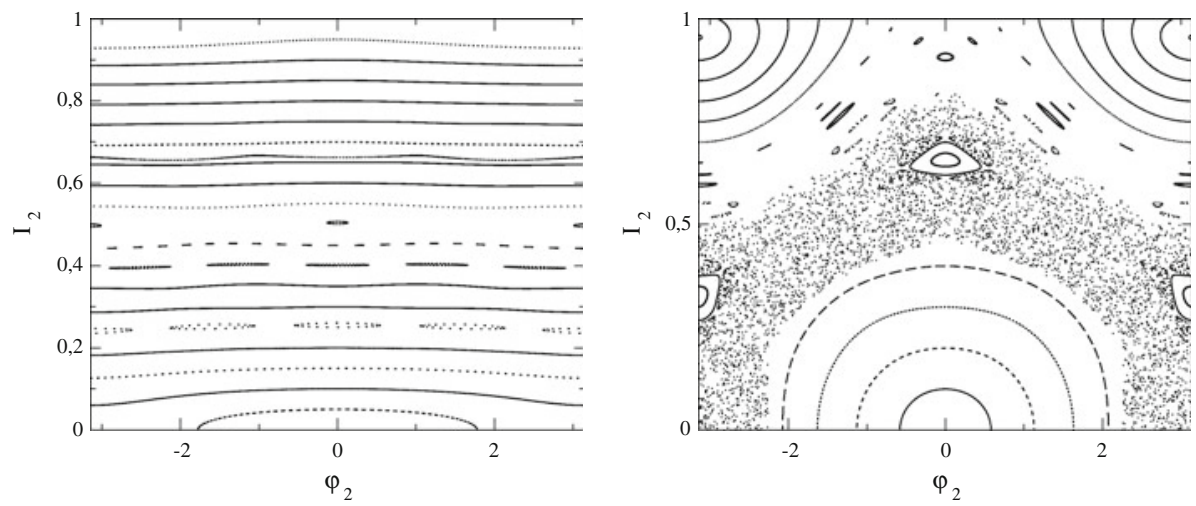

Fig. 1 Poincaré section of (3) for $\epsilon=0.01$ (left panel) and $\epsilon=0.4$ (right panel). On the right panel we see that the region of the phase space with $I_{2} \in[0,1]$ is characterized by the overlapping of resonances

In Sect. 5 we study the stable and unstable manifolds of $\Lambda$ itself. The existence of these stable and unstable manifolds follow from the normal hyperbolicity of $\Lambda$, which we establish with numerical tools. In fact, for generic quasi-integrable systems (see discussion in Guzzo et al. 2009a), the normal hyperbolicity of the invariant manifolds cannot be obtained by extension from the unperturbed case $\epsilon=0$, because for $\epsilon=0, \Lambda$ is not normally hyperbolic. We found numerical evidences of the normal hyperbolicity of $\Lambda$ with the methods introduced in Guzzo et al. (2009a,b), for some of the values of $\epsilon$ used in our numerical experiments, precisely for $\epsilon=10^{-5}, 10^{-4}, 10^{-3}, 10^{-2}$. We refer to Guzzo et al. (2009b) for all the technical details.

For small values of the perturbing parameter the Hamiltonian system (1) satisfies the hypotheses of the Nekhoroshev theorem. In the regime of validity of the Nekhoroshev theorem, normal forms with exponentially small remainder are valid in the resonant domains. More precisely, in a domain of the phase space characterized by the presence of only one resonant angle $k \cdot \varphi=k_{1} \varphi_{1}+\cdots k_{n} \varphi_{n}$ of order $|k|=\sum_{i=1}^{n}\left|k_{i}\right|$ smaller than a threshold $K \sim 1 / \epsilon^{\alpha}$, with suitable positive $\alpha$, the normal form is of the type:

$$
h(I)+\epsilon g_{k}(I, k \cdot \varphi)+R_{K}(I, \varphi)
$$

where the norm of the remainder $R_{K}$ is exponentially small with respect to $K$, and therefore with respect to $1 / \epsilon^{\alpha}$. We remark that the above Hamiltonian is integrable apart from the exponentially small remainder $R_{K}$, because it depends on only one angle $k \cdot \varphi$. Therefore, if the system:

$$
h(I)+\epsilon g_{k}(I, k \cdot \varphi)
$$

has an hyperbolic torus, its stable and unstable manifolds do not split, because the Hamiltonian is integrable. If the hyperbolic torus persists in the perturbed system (6), then the exponentially small remainder $R_{K}$ can be responsible of the splitting of these manifolds. It is known from the work of Neishtadt (1984) that the splitting of separatrices cannot exceed the size of the remainder of the normal form, therefore, the aim of this paper is to measure the exponential dependence of the splitting on the perturbing parameter. 


\section{Measure of the exponential splitting of the homoclinic tangle}

As it is well known (Simó 2000), the direct measure of exponentially small splittings is a difficult numerical task. In Simó and Valls (2001) the authors study analytically and numerically the splitting of two-dimensional hyperbolic tori in the classical example of Arnold diffusion, with two equal parameters. In this paper we also consider splitting of two dimensional tori, but with a more general perturbation which cannot be reduced to an Arnold like example. Our approach is different from the one used in Simó and Valls (2001). In fact we use the technique that we introduced in Lega et al. (2009) to measure the exponential splitting of a four dimensional maps. For this kind of measures one has to be aware of the propagation of round off errors in the dynamics. We have used a symplectic integrator and computed the evolution of the orbits both with double and quadruple precision checking that the result is invariant with respect to the used precision. This allows to say that the splitting is not due to numerical errors. When the limit of the double precision is attained the size of the splitting turns out to be different for the double and quadruple precision, scaling with $\epsilon$ for the higher precision computation and remaining constant with $\epsilon$, and therefore due to the numerical error for the lower precision computation. The exercise is then repeated by computing orbits with quadruple and octuple precision. In our simulation we didn't reach the limit of quadruple precision. The study of Hamiltonians systems allows for a comparison with the analytical and semi-analytical studies, which usually refer to the Hamiltonian case (such as Efthymiopoulos 2008) rather than the map case.

We consider an hyperbolic torus of frequency $\bar{\omega}$ on the normally hyperbolic invariant manifold $\Lambda$ (2). We compute a chaotic observable zone as follows: we consider a set of orbits with initial conditions $\left(I_{1}(0), I_{2}(0), \varphi_{1}(0), \varphi_{2}(0)\right)$ in a neighbourhoud of $\left(I_{1}, \varphi_{1}\right)=(0, \pi)$, with $\varphi_{2}(0), \varphi_{3}(0)=0$ and $I_{2}(0)$ is chosen so that the frequency of the angle $\varphi_{2}$ is $\bar{\omega}_{2}$. This frequency is numerically computed using a variant (Sidlichovský and Nesvorný 1997) of the Laskar et al. (1992) frequency analysis algorithm. ${ }^{2}$ We numerically compute the motions over a time $\bar{t}$ and we consider the Poincaré section on $\varphi_{3}$. Moreover, we only consider the points of the Poincaré section which satisfy the following 'slice cutting' condition on the variables $\varphi_{2}, I_{2}$ :

$$
\left|\varphi_{2}-\varphi_{2}(0)\right|<\sigma,\left|I_{2}-I_{2}(0)\right|<\sigma .
$$

The parameter $\sigma$ is chosen small enough that the set of points in the plane $\left(I_{1}, \varphi_{1}\right)$ fills a region which does not shrink for further reductions of $\sigma$ itself: that is, by reducing $\sigma$, one reduces the density of points, but not the size of the region filled by these points. With such a technique which combines Poincaré section and slice cutting, we obtain a chaotic zone in the plane $\left(I_{1}, \varphi_{1}\right)$ which is associated to the unstable manifold of the hyperbolic torus of frequency $\bar{\omega}_{2}$. Hereafter, we will call it: Chaotic Hyperbolic Section (CHS).

We expect that in the regime of validity of the Nekhoroshev theorem the size of the chaotic zone depends on the distance $d$ from the hyperbolic point and on $\epsilon$ approximately like:

$$
L(d, \epsilon)=f(d) \epsilon^{a} \exp \left(-\left(\frac{\epsilon^{*}}{\epsilon}\right)^{\alpha}\right)
$$

where the function $f$ and the constants $a, \epsilon^{*}, \alpha$ are unknown and will be determined by a numerical fit.

$\overline{2}$ The routines can be found on the website: http://www.boulder.swri.edu/ davidn/fmft/fmft.html. 

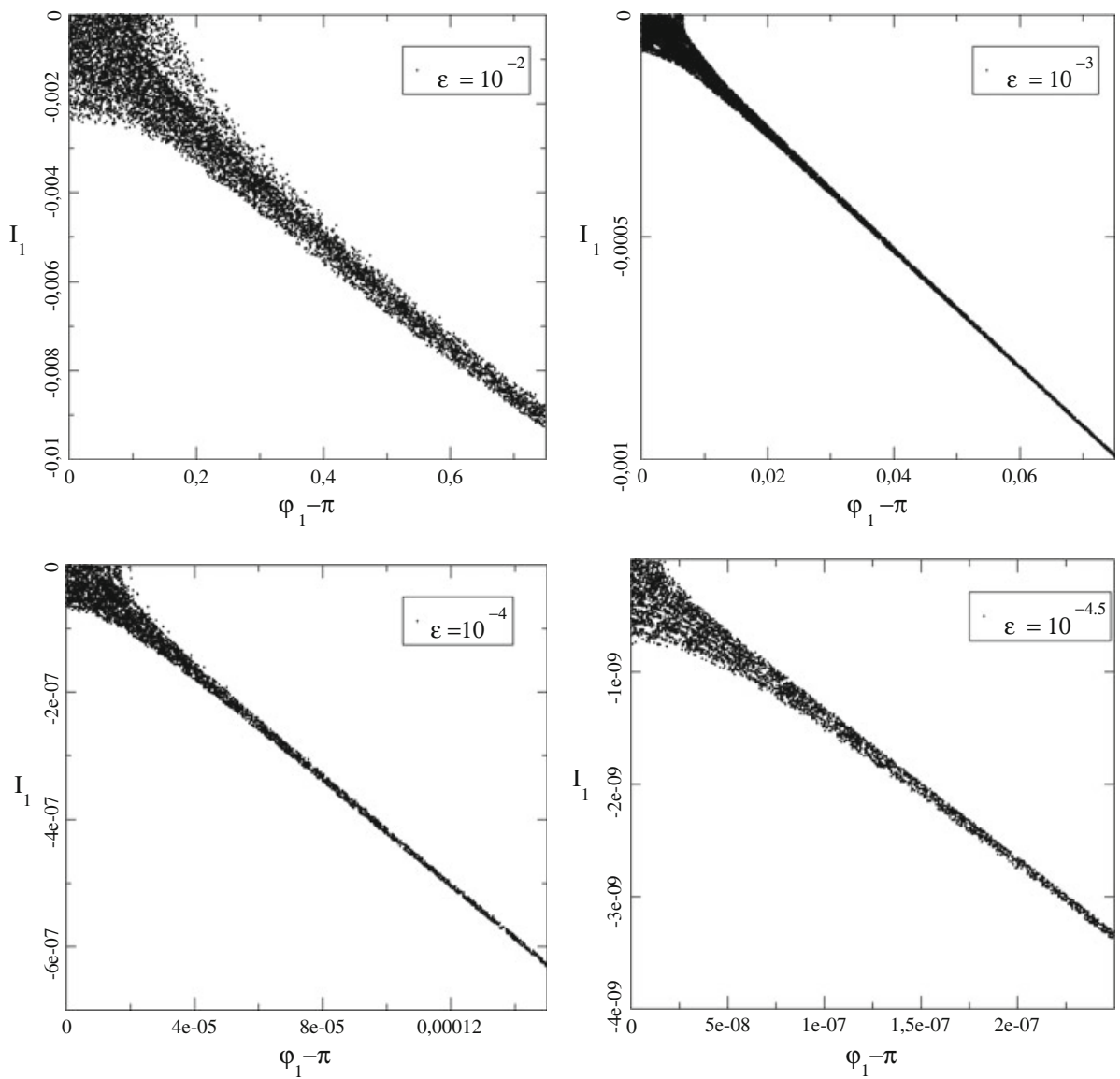

Fig. 2 Detection of the CHS associated to the unstable manifold of the hyperbolic torus with frequency $\bar{\omega}_{2}=\frac{(\sqrt{5}-1)}{2}$ for different values of $\epsilon$ going from $10^{-2}$ down to $10^{-4.5}$. The values of the action $I_{1}$ have been rescaled by a factor $\sqrt{10^{-3} / \epsilon}$. The zoom out around the origin from the panel at the top left to the panel at the bottom right allows one to appreciate visually the strong decreasing of the size of the chaotic zone as a function of $\epsilon$

Figure 2 shows the CHS obtained for a set of $N=100$ orbits, $\sigma=10^{-4}$, for different values of $\epsilon$. The integration time $\bar{t}$ and the interval $\Delta \varphi_{1}$ for the variable $\varphi_{1}(0)$ are suitably chosen for each value of $\epsilon$ and reported in Table 1 .

The zooms out around the origin, from the panel at top left to the panel at bottom right, illustrate visually the strong decrease of the size of the chaotic zone associated to the unstable manifold by changing the perturbing parameter. The form of (8) allows to study separately the variation of $L$ with respect to its variables. For instance, we measure the quantity $L_{d}(\epsilon)$ at a distance $d$ from $\varphi_{1}=\pi$ as the difference between the maximum and the minimum of the actions $I_{1}$ of the points filling the CHS. In order to obtain a numerical evidence of the exponential character of $S(\epsilon)=L_{d}(\epsilon) / f(d)$ we cannot simply fix $d$ and measure $L_{d}(\epsilon)$ by changing $\epsilon$, because, for fixed $d$, we would be able to measure effectively the amplitude of the chaotic zone only through a small interval in $\epsilon$. In fact, due to the strong decreasing of the amplitudes with $\epsilon$, 
Table 1 Parameters for the detection of the CHS associated to the unstable manifold of the hyperbolic torus with frequency $\bar{\omega}_{2}=\frac{(\sqrt{5}-1)}{2}$

\begin{tabular}{lll}
\hline$\epsilon$ & $\bar{t}$ & $\Delta \varphi_{1}$ \\
\hline $10^{-4.5}$ & $10^{9}$ & $10^{-15}$ \\
$10^{-4}$ & $5 \times 10^{8}$ & $10^{-13}$ \\
$10^{-3}$ & $10^{8}$ & $10^{-12}$ \\
$10^{-2}$ & $10^{7}$ & $10^{-10}$
\end{tabular}

The amplitude of the neighbourhoud of $\varphi_{1}=\pi$ is denoted by $\Delta \varphi_{1}$

from Fig. 2 it appears clearly that each chaotic zone is detected up to a maximal distance $d_{\max }$ from $\varphi_{1}=\pi$ which reduces drastically with $\epsilon$. In order to obtain a numerical measure of $S(\epsilon)=L_{d}(\epsilon) / f(d)$ through many orders of magnitude in $\epsilon$ we need therefore a numerical fit for $f(d)$, so that, for any $\epsilon$, we can suitably choose the distance $d$.

We have fitted the function $f(d)$ using the numerical computation of $L_{\epsilon}(d)$ as a function of the distance $d$ and repeating the measure for different values of $\epsilon$ as we did in Lega et al. (2009). Each data set is well fitted by a power law: $d^{m_{\epsilon}}$, and the set of the slopes $m_{\epsilon}$ has mean value $\bar{m}=-1.1$ and standard deviation 0.05 . We will consider the function $d^{\bar{m}}$ as a good fit of $f(d)$ in (8).

Figure 3 top, left shows the logarithm of $1 / S$ as a function of the logarithm of $1 / \epsilon$. The exponential dependence of $S$ on $\epsilon$ appears clearly.

Thanks to the fact that the measure spans 2.5 orders of magnitude in $\epsilon$, we could fit the parameter $\alpha$ as follows:

$$
\log (1 / S)=a x+b 10^{\alpha x} \quad x=\log (1 / \epsilon)
$$

In Fig. 3 top, right we plot the $\log (1 / S)$ as a function of $1 / \epsilon^{\alpha}$ for the fitted value: $\alpha=0.27$.

The linear behaviour expected from (9) appears clearly. Moreover, for a convex system with $n$ degrees of freedom, the remainder $R_{K}$ is estimated smaller than the exponential of $K \sim 1 / \epsilon^{\alpha}$, with $\alpha=1 / 2(n-d)$ (this estimate has been proved in Pöschel (1993) and Lochak (1992), following an idea of Benettin and Gallavotti (1986)) in the neighbourhoud of a fixed resonance, generated by $d$ independent resonances, that is $\alpha=1 / 4$ in the case under study which is very close to the fitted value $\alpha=0.27$.

However, we must point out that the determination of $\alpha$ is quite delicate: the plot of $\log (1 / S)$ with respect to $1 / \epsilon^{\alpha}$ with $\alpha=0.2$ (Fig. 3 bottom, left) and $\alpha=0.3$ (Fig. 3 bottom, right) show a slight departure from the linear behaviour between $\log (1 / S)$ and $1 / \epsilon^{\alpha}$. We can conclude that $0.2<\alpha<0.3$, which means that the digit 2 is well determined, while the following one is uncertain. To restrict the confidence interval for $\alpha$ we should go to smaller values of $\epsilon$ which means very long integrations (more than $t=10^{9}$ ), at higher and higher precision computations. This is beyond the purpose of the present paper.

We have repeated the experience, measuring the size of the CHS for the Hamiltonian (1) for the resonance $I_{1}=2 I_{2}$ for which Efthymiopoulos (2008) provides a semi-analytic computation of the size of the remainder. The choice of initial conditions is a more difficult task than for the resonance $I_{1}=0$ and needs a preliminary detection of the resonant line. Precisely, we have computed the Fast Lyapunov Indicator (FLI hereafter, see Froeschle et al. (2000) and Sect. 5 in this paper) on a set of $500 \times 500$ initial conditions regularly spaced with respect to the variables $I_{1}-2 I_{2}$ and $2 I_{1}+I_{2}$, with $\varphi_{1}=\pi$ and $\varphi_{2}=0$. The resonance 

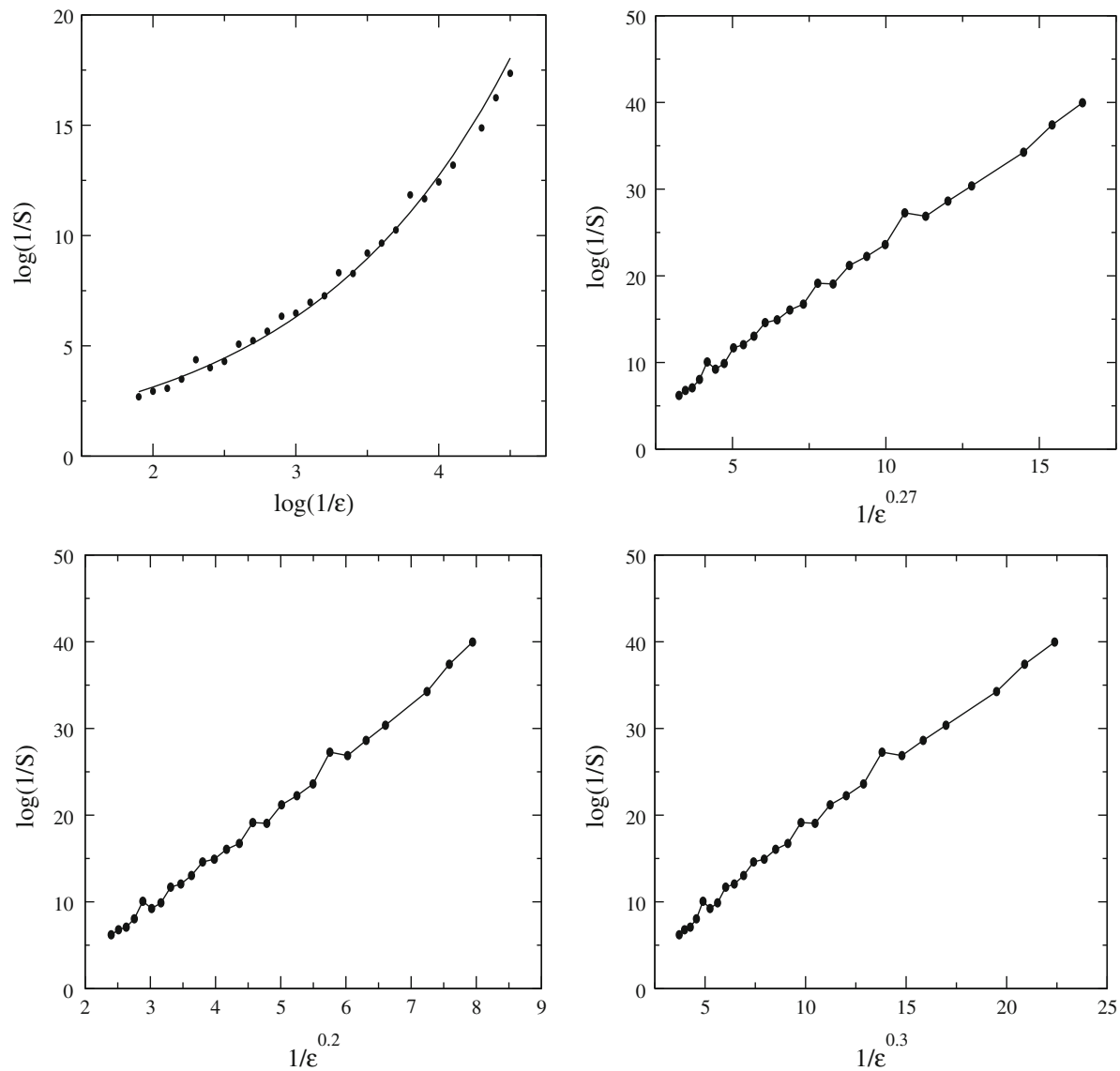

Fig. 3 Variation of $S(\epsilon)$ with respect to $\epsilon$. Top left the exponential character of the relation between $S(\epsilon)$ and $\epsilon$ in agreement with (8) appears clearly. The continuous line corresponds to the exponential fit. Top right The plot of $\log (1 / S)$ with respect to $1 / \epsilon^{\alpha}, \alpha=0.27$ confirms the linear behaviour expected form (9). In order to determine a confidence interval to the fitted $\alpha$ we also plot the $\log (1 / S)$ with respect to $1 / \epsilon^{\alpha}$ with $\alpha=0.2$ (Bottom left) and $\alpha=0.3$ (Bottom right)

$I_{1}=2 I_{2}$ clearly appears as a light grey line (Fig. 4 left, panel) in the center of the FLI-chart. Thanks to the detection of the resonant line we have chosen chaotic initial conditions for the $I_{1}-2 I_{2}$. The other initial conditions are: $2 I_{1}(0)+I_{2}(0)=0.76 \varphi_{1}(0)=\pi$ and $\varphi_{2}(0)=0$. The CHS, providing the measure of $L_{d}(\epsilon)$, is then obtained by computing a set of $N=500$ orbits and plotting $I_{1}-2 I_{2}$ versus the resonant angle $\varphi_{1}-2 \varphi_{2}$ for $\sigma=510^{-3}$, (Fig. 4, right, panel). We have obtained for the function $f(d): f(d) \simeq d^{-1.05}$. In order to compare to the results of Efthymiopoulos (2008) we have fitted $S(\epsilon)=L_{d}(\epsilon) / f(d)$ with the following law: $S(\epsilon) \simeq \exp \left(-b / \epsilon^{\alpha}\right)$ (Fig. 5) the best fit of $\alpha$ providing the value $\alpha=0.25$. When comparing with Fig. 6 of Efthymiopoulos (2008) we remark that the exponential fit with our numerical method holds on the whole interval of measure while in Efthymiopoulos (2008) a power law fit between the remainder and the perturbing parameter is obtained on the larger values of $\epsilon\left(10^{-2}<\epsilon<10^{-1}\right)$ while the fit is exponential for $10^{-4}<\epsilon<10^{-2}$ with a value of $\alpha=0.21$ compatible with our result. 

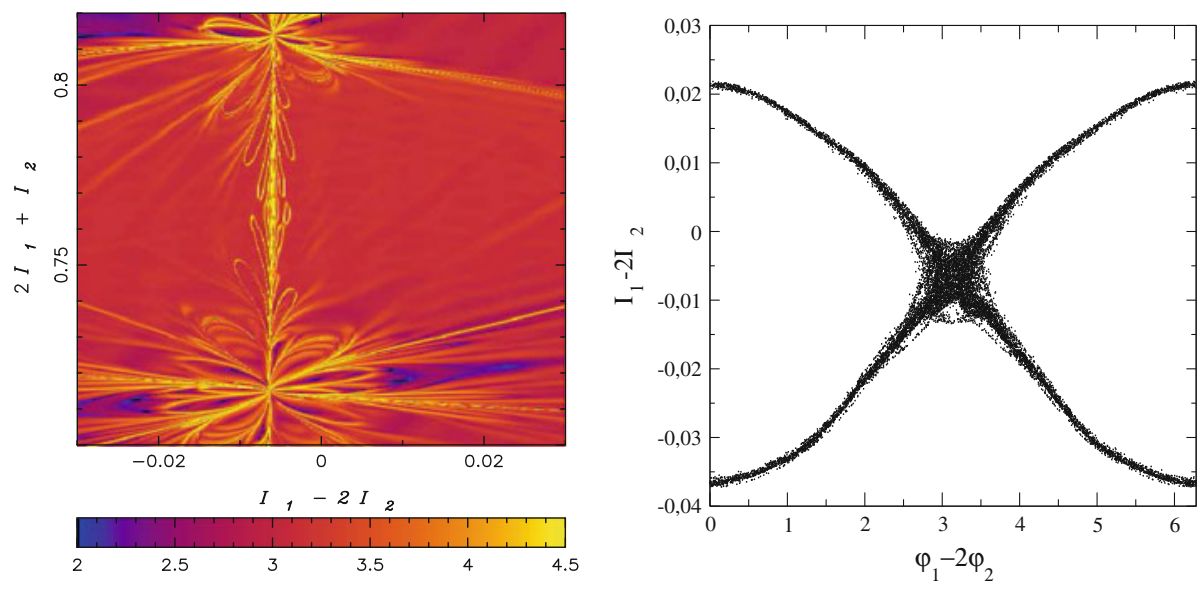

Fig. 4 Left panel FLI-chart for a set of $500 \times 500$ initial conditions regularly spaced $I_{1}-2 I_{2}$ and in $2 I_{1}+I_{2}$ with $\varphi_{1}=\pi$ and $\varphi_{2}=0$. The integration time is $t=1000$ and the perturbing parameter is $\epsilon=0.07$. The color scale range from 2 (dark grey, corresponding to regular resonant islands) to 4.5 (light grey corresponding to chaotic orbits). The grey background $(F L I \simeq 3$ ) stands for invariant tori. Right panel Detection of the CHS associated to the hyperbolic torus with $2 I_{1}(0)+I_{2}(0)=0.76$ for $\epsilon=0.07$. The values of $I_{1}-2 I_{2}$ versus the resonant angle $\varphi_{1}-2 \varphi_{2}$ are plotted for $\sigma=510^{-3}$

Fig. 5 Variation of $S(\epsilon)$ with respect to $\epsilon$ for the resonance $I_{1}=2 I_{2}$. The exponential dependence of $S(\epsilon)$ with respect to $\epsilon$ appears clearly

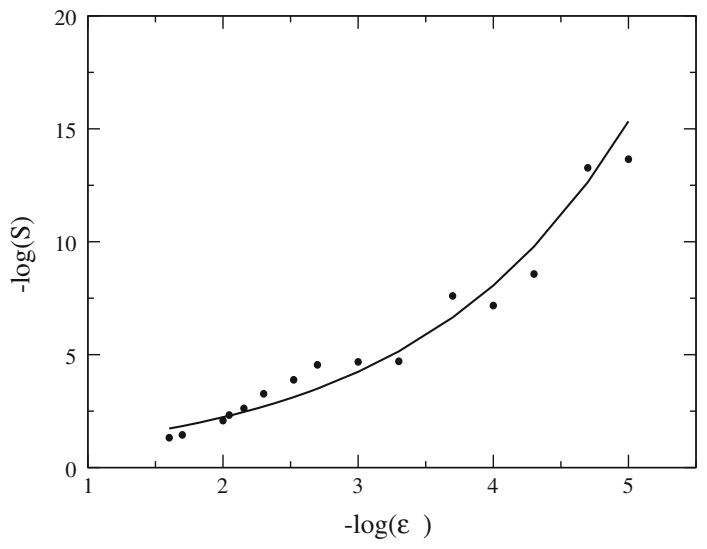

\section{On the connection between the remainder and diffusion}

In several papers (Lega et al. 2003; Guzzo et al. 2005, 2009a; Froeschlé et al. 2005) we numerically computed the diffusion coefficient $D$ in specific resonances, at different values of $\epsilon$. The intervals of $\epsilon$ spanned by these explorations were sufficiently large to conclude that $D(\epsilon)$ decreased faster than a power law, but were not so large to allow us to perform good exponential fits, as it is clearly explained in Froeschlé et al. (2005). Instead, the measures of the size of homoclinic tangles done in this paper and in Lega et al. (2009) are so large to allow us to perform exponential fits.

The problem of the connection between the remainder and the diffusion coefficient has been studied in detail in Efthymiopoulos (2008). Using the Hamiltonian model (1) the author shows that the diffusion coefficient $D$ reported in Lega et al. (2003) and the optimal remainder 
Fig. 6 Computation of the diffusion coefficient $D$ for different values of $\epsilon$ for the resonance $I_{1}=0$. Depending on the value of $\epsilon$, we used data sets of $N=100$ or $N=1000$ orbits and total integration times from $t=10^{7}$ up to $t=510^{9}$. The computed diffusion coefficient is well fitted by three different power laws $D(\epsilon) \simeq \epsilon^{m}$ with respectively $m 1=5.1, m 2=9.1$ and $m 3=14.5$ in different intervals of $\epsilon$

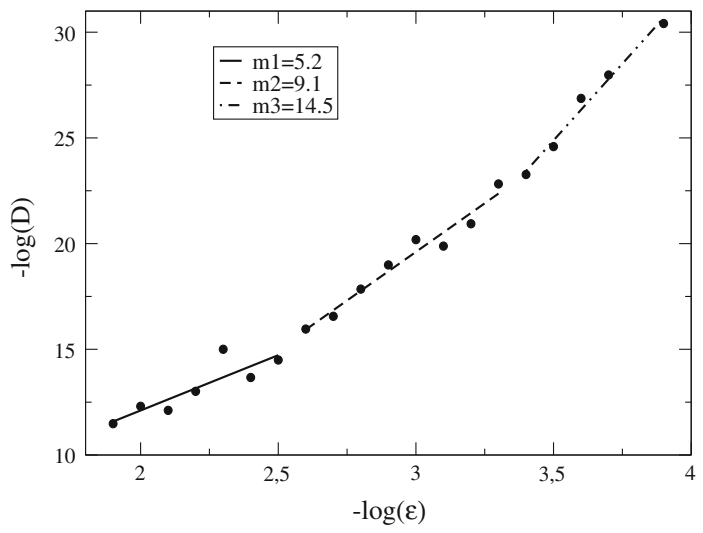

$R$ of the normal form constructed using a computer-assisted technique scales as $D \propto R^{3}$. The aim of this section is to find the relation between $S$ and $D$ for both resonances: $I_{1}=0$ and $I_{1}=2 I_{2}$. For the resonance $I_{1}=2 I_{2}$ we use the computation of the diffusion coefficient already published in Lega et al. (2003), while for the resonance $I_{1}=0$ we needed to compute $D$ with a new numerical experiment. Using the same technique that was developed in several papers (Lega et al. 2003; Guzzo et al. 2005), we computed $D$ as follows. Denoting with $I_{2}^{j}(0), j=1, \ldots, N$ the initial conditions of a set of $N$ orbits and with $I_{2}^{j}(t)$ the corresponding values at time $t$, we compute the quantity:

$$
\bar{d}(n \delta t)=\frac{1}{M_{n}} \sum_{j:\left(\left|\varphi_{1}^{j}(t)-\pi\right| \leq \sigma_{1},\left|\varphi_{2}^{j}(t)\right| \leq \sigma_{2}\right)}\left(I_{2}^{j}(t)-I_{2}^{j}(0)\right)^{2}
$$

where $M_{n}$ is the number of points of the orbits satisfying the double slice cutting condition on the angles for for $t$ in the interval $(n-1) \delta t \leq t \leq n \delta t$. Then, we compute the diffusion coefficient $D$ as the slope of the best linear fit of the quantity $\bar{d}(n \delta t)$ for $\sigma_{j}=10^{-3}, j=1,2$ and $\delta t=10^{6}$. The initial values for the sets of diffusing orbits are chosen (as in Sect.3): $\varphi_{2}(0)=0 ; I_{2}(0)$ such that the frequency of $\varphi_{2}$ is $0.5(\sqrt{5}-1) ; \varphi_{1}(0)=\pi$ and $-10^{-12}<$ $I_{1}<10^{-12}$. Figure 6 shows the variation of the diffusion coefficient for the resonance $I_{1}=0$ as a function of $\epsilon$. In agreement with the results of Lega et al. (2003), Guzzo et al. (2005), we find that $D(\epsilon)$ can not be fitted to a single power law in $\epsilon$, while we fitted the data to three different power laws $D(\epsilon) \simeq \epsilon^{m}$ with respectively $m 1=5.1, m 2=9.1$ and $m 3=14.5$ in different intervals of $\epsilon$.

This is sufficient to exclude a global power law and the changes of the local slopes are in agreement with the expected exponential decrease of $D$.

Let us remark that $D$ is obtained as an average. We are aware of the fact that points in the chaotic zone can have different behavior. We have introduced in Guzzo et al. (2009b) a criterium of "good" statistic when the diffusing points have a regular behavior. For an important class of systems called "a priori unstable" 3 we have obtained that, for values of the perturbing parameter lower than a critical value, the statistic is "good" and the diffusion coefficient obtained as an average could be used to do predictions. The statistic is less good for higher values of the perturbing parameter and we observe a less regular behavior of the diffusing orbits, due mainly to the effect of secondary resonances, but the mean value $D$ is still

3 A quasi-integrable Hamiltonian system is said to be "a priori unstable" if it has an hyperbolic manifold in its unperturbed part. 

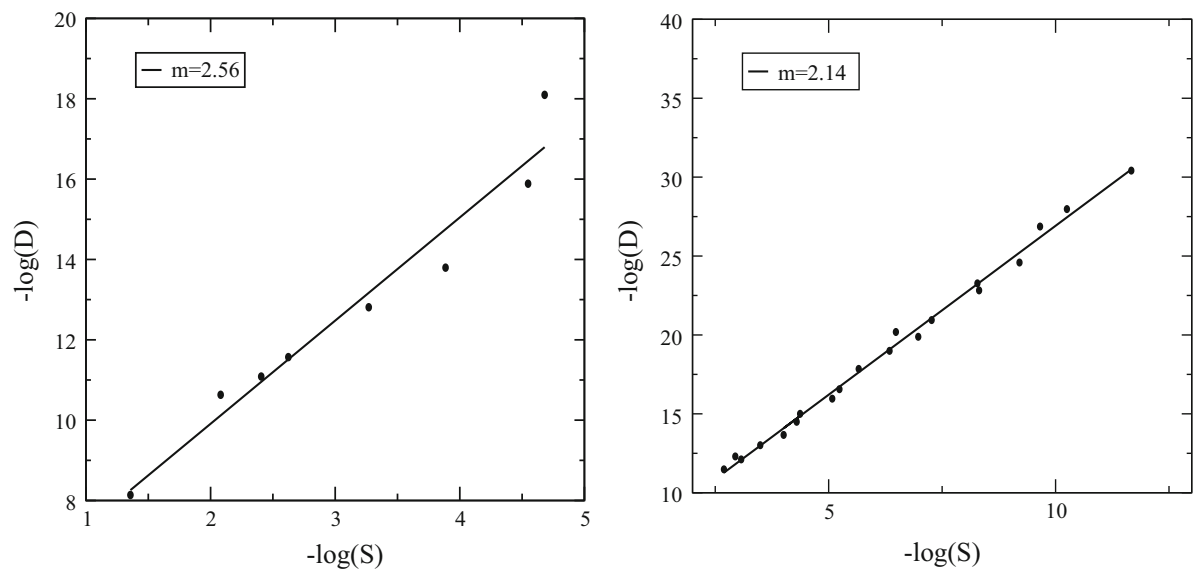

Fig. 7 (Left panel) The diffusion coefficient $D$ as measured in Lega et al. (2003) is plotted as a function of the size of the chaotic zone. The best linear fit gives: $D \propto S^{2.5}$.(Right panel) The diffusion coefficient $D$ computed for the resonance $I_{1}=0$ plotted as a function of the size of the chaotic zone. The best linear fit gives: $D \propto S^{2.14}$

representative of diffusion and the error on $D$ measures the effect of secondary resonances. Actually, as we have shown in Guzzo et al. (2009b), while the numerical error affects the individual integrated orbits after some Lyapunov times, it affects much less the computation of statistical quantities such as the Lyapunov exponents and the diffusion coefficients. The model studied in the present paper belongs to the class of the so-called "a priori stable" systems $^{4}$, for which the effect of secondary resonances is important for any value of $\epsilon$. Again we can consider that the mean value $D$ is representative of diffusion.

In the interval of $\epsilon$ for which we could measure the diffusion coefficient Fig. 7 shows a correlation of the kind $D \propto S^{2.5}$ between the diffusion coefficient and the size of the chaotic zone for the resonance $I_{1}=2 I_{2}$ and $D \propto S^{2.1}$ for the resonance $I_{1}=0$. We remark that the interval in $\epsilon$ is sufficiently large to conclude that both $D(\epsilon)$ and $S(\epsilon)$ decrease faster than a power law. We have worked on values of $\epsilon$ below the threshold of transition to the Chirikov's overlapping of resonances regime, and we have found results compatible with an exponential regime on the whole interval of measure. When comparing with Efthymiopoulos (2008) we have a clear disagreement for the larger values of $\epsilon, \epsilon>0.01$. The difference lies probably on the methods used: on the one hand we have a pure numerical method on the whole model, on the other hand we have a semi-analytical tool whose validity is certainly improved for values of $\epsilon$ well below the threshold of transition to the Chirikov's regime. Therefore, we claim that our results on both the diffusion coefficient and the measure of the size of the remainder are in agreement with Nekhoroshev theorem.

\section{Topology of the hyperbolic invariant manifold}

Thanks to the FLI method, it is possible to compute the stable and unstable manifolds of the invariant manifold $\Lambda$, not only of an hyperbolic torus (see Guzzo et al. 2009a). In Guzzo et al. (2009a) we have provided for the first time a detailed description of the topology of the

4 A quasi-integrable Hamiltonian system is said to be "a priori stable" if no hyperbolic manifolds exist in the unperturbed part. 
stable (unstable) manifolds of quasi-integrable systems with $n=2$ degrees of freedom. We have shown that the topology of the stable (unstable) manifolds presents peculiar flower-like structures which we explained by using a model of weakly interacting resonances. Using the mapping studied also in Lega et al. (2009) we have detected transitions in this topology which are related to the local predominant resonances identified with the local rational approximations of the frequencies, and we have shown a correlation among these transitions and the Arnold diffusion.

In this section we show that these correlations occur also for the Hamiltonian model (1) considered so far, therefore supporting their generic character. We remark that, using the Melnikov method for the computation of the separatrix splitting a relation between the size of the splitting and the dominant harmonics in models that are close to the Arnold's one was provided in Simó (1994), Delshams et al. (1997). As previously said, we work with a more general perturbation that cannot be reduced to that of the Arnold example so that Melnikov method cannot be directly used.

With our model (1) we compute the intersection between the stable and unstable manifolds of $\Lambda$ with the surface $S$ defined by:

$$
S=\left\{\left(I_{1}, I_{2}, \varphi_{1}, \varphi_{2}\right):\left(\varphi_{1}, \varphi_{2}\right)=(\pi, 0)\right\}
$$

We recall that for $\epsilon=0$, any initial condition $\left(I_{1}, I_{2}\right)$ on $S$ does not return on $S$, or it returns exactly on $\left(I_{1}, I_{2}\right)$. Therefore, each invariant torus, is represented symbolically by the initial condition of the torus belonging to $S$. If $\epsilon \neq 0$ the subsisting invariant tori intersect transversely $S$ only on one point, and therefore each invariant torus is still symbolically represented by a point on $S$. The section $S$ contains possibly many points belonging to invariant tori, but these tori do not trap motions in the 3 degrees of freedom Hamiltonian: there is the possibility of diffusion even for very small $\epsilon$. This diffusion may be supported by stable and unstable manifolds, and therefore we compute the intersections $S_{u}^{*}=S \cap W_{u}, S_{s}^{*}=S \cap W_{s}$, where $W_{s}$ and $W_{u}$ are the stable and unstable manifolds of $\Lambda$.

Using the technique introduced in Guzzo et al. (2009a) we use the FLI to numerically detect the sets $S_{u}^{*}, S_{s}^{*}$. Precisely, we compute the FLI for a grid of points of $S$ up to some given time $T$ : the points of the grid which have the highest values of the FLI are those points whose orbit approaches an hyperbolic invariant manifold within the computation time $T$, because the growth of tangent vectors is bigger near the hyperbolic manifolds. Therefore, a short-time computation of the FLI allows one to detect a neighborhood of a finite piece of the stable manifold (for more details the reader can refer to Guzzo et al. (2009a)).

\subsection{Topology of the hyperbolic manifolds for the resonance $I_{1}=0$}

To help us in the identification of the dominating structures, as it was done in Guzzo et al. (2009a), we compare the location of the flower like structures to the approximating sequence of the frequency. Precisely, in the integrable approximation, the resonances intersect the invariant manifold $\Lambda$ in:

$$
\left(I_{1}, I_{2}\right)=\left(0, \frac{k_{1}}{k_{2}}\right),
$$

with $k_{1}, k_{2} \in \mathbb{Z}$. Therefore, in the neighborhood of $\left(I_{1}, I_{2}\right)=(0,0.5(\sqrt{5}-1))$, we expect to find flower-like structures centered around the points (12), with $k_{1}, k_{2}$ defined by the main approximations of golden number $(0.5(\sqrt{5}-1))$. 

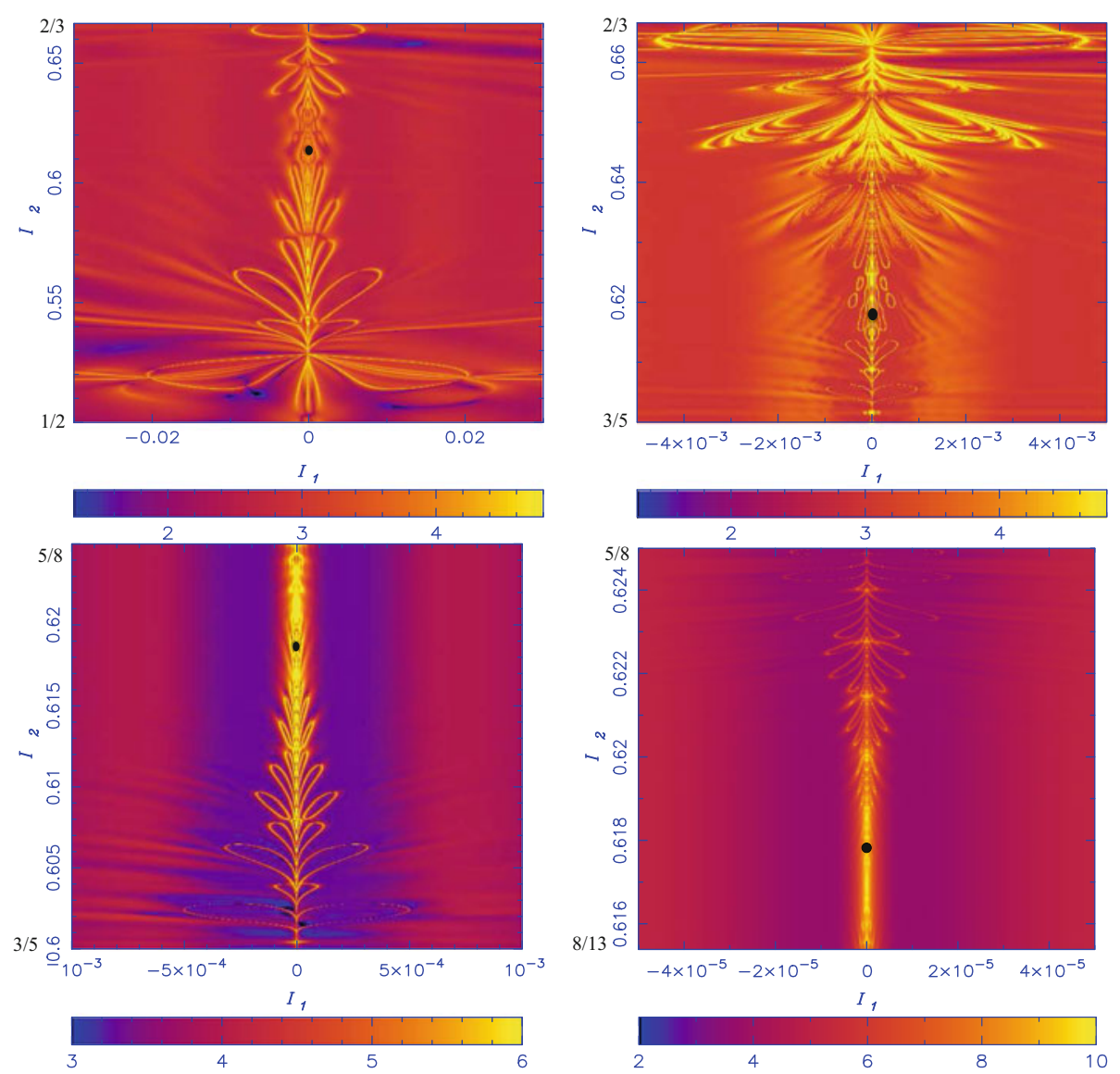

Fig. 8 Computation of the FLI for $\epsilon=0.01,0.004$ (on the top for $T=400$ ) and $\epsilon=0.001,0.0003$ (on the bottom) for respectively $T=2000$ and $T=4000$. The light grey gives the points of the grid approaching an hyperbolic manifold within the computational time T. In each panel the color scale range is chosen in order to enhance the structure of the detected neighborhood of the stable manifold. The black dot indicates the point $\left(I_{1}, I_{2}\right)=(0,0.5(\sqrt{5}-1))$

The first points of the approximating sequence are given by the well known Fibonacci sequence:

$$
I_{2}=1, \frac{1}{2}=0.5, \frac{2}{3} \sim 0.6666, \frac{3}{5}=0.6, \frac{5}{8}=0.625, \frac{8}{13} \sim 0.6154 \ldots
$$

Figure 8 shows the results of the FLI computation on a grid of $1000 \times 1000$ initial conditions regularly spaced on $S$ for different values of $\epsilon$ and different integration times $T$. The size of each picture on $I_{2}$ is chosen according to the successive values of the Fibonacci sequence (13), for instance the top left panel has top value $I_{2}=2 / 3$ and bottom value $I_{2}=1 / 2$ (as indicated); and we have suitably shrinked the interval around $I_{1}=0$. The point $\left(I_{1}, I_{2}\right)=(0,0.5(\sqrt{5}-1))$ is represented by a black dot.

We start describing Fig. 8 top, left. We observe a flower-like structure at the bottom of the picture corresponding to the intersection of $I_{1}=0$ with $I_{2}=1 / 2$. We also observe "petals" 
top oriented along $I_{1}=0$. As shown in Guzzo et al. (2009a) the "petals" are generated at the exact resonance $(0,1 / 2)$ and belong to the stable manifold of the single resonance $I_{1}=0$. At the top of the picture we observe another flower-like structure originated at the point $I_{1}=0, I_{2}=2 / 3$ with petals down oriented. The point $\left(I_{1}, I_{2}\right)=(0,0.5(\sqrt{5}-1))$ is located close to the intersection between the two petals structures. Therefore the corresponding value of $\epsilon=0.01$, is close to a transition value: the resonance $I_{2}=1 / 2$ dominates the local dynamics for $\epsilon>0.01$ while $I_{2}=2 / 3$ is the local dominant resonance for $\epsilon \leq 0.01$.

We have repeated the procedure for different values of $\epsilon$ and we have found the three different cases:

(i) for $3 \times 10^{-3}<\varepsilon \leq 0.01$ the dominant flower-like structure around the point $\left(I_{1}, I_{2}\right)=$ $(0,0.5(\sqrt{5}-1))$ is centered on $I_{2}=2 / 3$. For $\epsilon=0.004$ (top right panel of Fig. 8) one can appreciate that the petals around the point $\left(I_{1}, I_{2}\right)=(0,0.5(\sqrt{5}-1))$ are still centered on $\left(I_{1}, I_{2}\right)=(0,2 / 3)$ (top of the picture). Moreover the two values of $\epsilon$ of Fig. 8, top correspond to the lowest and higher value of $\epsilon$ for which the relation $D(\epsilon) \simeq \epsilon^{5.2}$ is valid.

(ii) For $3 \times 10^{-3} \leq \epsilon \leq 5 \times 10^{-4}$ we found that the dominant flower-like structure is originated at $I_{2}=3 / 5$ (bottom of the picture), which is the fourth Fibonacci approximating (13). In Fig. 8, bottom left panel, we report the computation of the FLI for $\epsilon=3 \times 10^{-3}$ and $T=2000$. We remark that the resonance $I_{2}=3 / 5$ dominates the dynamics in the interval of $\epsilon$ for which the diffusion coefficient behaves as $D(\epsilon) \simeq \epsilon^{9.1}$.

(iii) For $4 \times 10^{-4} \leq \epsilon<10^{-4}$ we found that the dominant flower-like structure is originated at $\left(I_{1}, I_{2}\right)=(0,5 / 8)$, which is the fifth point of the approximating sequence (13). In Fig. 8 bottom,right we report the computation of the FLI for $\epsilon=3 \times 10^{-4}$ and $T=4000$. The resonance $I_{2}=5 / 8$ dominates the dynamics in the interval of $\epsilon$ for which the diffusion coefficient behaves as $D(\epsilon) \simeq \epsilon^{14.5}$.

This study shows that the intervals of $\epsilon$ corresponding to the different dominating topological structures are correlated to the changes of slope of the diffusion coefficient. This fact can be related to the construction of the resonant normal forms in the proof the Nekhoroshev theorem, where different domains of the action space are related to different resonances according to the value of $\epsilon$.

\section{Conclusion}

Using a 3 degrees of freedom Hamiltonian system as a model problem we have measured through different orders of magnitude in $\epsilon$ the size of the chaotic zone corresponding to the remainder of the normal form. With a method of computation introduced in Lega et al. (2009) we have obtained an exponential behaviour for two different resonances. The obtained value of the parameter $\alpha$ characterizing the exponential law is close to that expected from the Nekhoroshev theorem and in agreement with the result found in Efthymiopoulos (2008). We have also obtained a good correlation between the diffusion coefficient and the size of the homoclinic tangle. Moreover, the study of the topology of normally hyperbolic manifolds shows transitions related to the local dominant resonances, which are different at different values of $\epsilon$. Such transitions are related to the changes of the diffusion properties around the resonance. As a conclusion we have three different kinds of measure: the size of the homoclinic tangle, the diffusion coefficient and the topology of hyperbolic manifolds which turn out to be correlated and in agreement with the Nekhoroshev theorem. 


\section{References}

Arnold, V.I.: Small denominators and problems of stability of motion in classical and celestial mechanics. Russ. Math. Surveys 18, 85-191 (1963)

Arnold, V.I.: Instability of dynamical systems with several degrees of freedom. Sov. Math. Dokl. 6, 581585 (1964)

Benettin, G., Gallavotti, G.: Stability of motions near resonances in quasi-integrable Hamiltonian systems. J. Stat. Phys. 44, 293-338 (1986)

Berti, M., Bolle, P.: Fast Arnold's diffusion in systems with three time scales. Discret. Continuous Dyn. Syst. Ser. A 8(3), 795-811 (2002)

Bessi, U.: An approach to Arnold diffusion through the calculus of variations. Nonlinear Anal. T. M. A. 26, 1115-1135 (1996)

Bessi, U.: Arnold's examples with three rotators. Nonlinearity 10, 763-781 (1997)

Bessi, U., Chierchia, L., Valdinoci, E.: Upper bounds on Arnold diffusion time via mather theory. J. Math. Pures Appl. 80, 105-129 (2001)

Berti, M., Biasco, L., Bolle, P.: Drift in phase space: a new variational mechanism with optimal diffusion time. J. de mathématiques pures et appliquées 82, 613-664 (2003)

Celletti, A., Chierchia, L.: KAM stability for a three-body problem of the solar system. Z. Angew. Math. Phys. 57(1), 33-41 (2006)

Celletti, A., Chierchia, L.: KAM stability and celestial mechanics. Mem. Amer. Math. Soc. 187, 878 (2007)

Chierchia, L., Gallavotti, G.: Drift and diffusion in phase space, Annales de l'IHP, section Physique Théorique, 60, 1994, pp. 1-144, see also Erratum in Vol. 68, 135 (1998)

Delshams, A., Gelfreich, V., Jorba, À., Seara, T.M.: Exponentially small splitting of sepatrices under fast quasiperiodic forcing. Commun. Math. Phys. 189, 35-71 (1997)

Efthymiopoulos, C.: On the connection between the Nekhoroshev theorem and Arnold diffusion. Celest. Mech. Dyn. Astron. 102, 49-68 (2008)

Froeschlé, C., Guzzo, M., Lega, E.: Local and global diffusion along resonant lines in discrete quasi-integrable dynamical systems. Celest. Mech. Dyn. Astron. 92(1-3), 243-255 (2005)

Froeschlé, C., Guzzo, M., Lega, E.: Graphical evolution of the Arnold web: from order to chaos. Science 289(5487), 2108-2110 (2000)

Guzzo, M.: Nekhoroshev stability of quasi-integrable degenerate Hamiltonian systems. Regul. Chaotic Dyn. 4(2), 78-102 (1999)

Guzzo, M.: The web of three-planet resonances in the outer solar system. Icarus 174, 273-284 (2005)

Guzzo, M.: The web of three-planet resonances in the outer solar system II. A source of orbital instability for Uranus and Neptune. Icarus 181, 475-485 (2006)

Guzzo, M., Morbidelli, A.: Construction of a Nekhoroshev like result for the asteroid belt dynamical system. Cel. Mech. Dyn. Astron. 66, 255-292 (1997)

Guzzo, M., Knežević, Z., Milani, A.: Probing the Nekhoroshev stability of asteroids. Cel. Mech. Dyn. Astron. 83, 121-140 (2002)

Guzzo, M., Lega, E., Froeschlé, C.: A numerical study of the topology of normally hyperbolic invariant manifolds supporting Arnold diffusion in quasi-integrable systems. Physica D 238, 1797-1807 (2009a)

Guzzo, M., Lega, E., Froeschlé, C.: A numerical study of Arnold diffusion in a priori unstable systems. Commun. Math. Phys. 290, 557-576 (2009b)

Guzzo, M., Lega, E., Froeschlé, C.: First Numerical Evidence of Arnold diffusion in quasi-integrable systems. DCDS B 5(3), (2005)

Laskar, J., Froeschlé, C., Celletti, A.: The measure of chaos by the numerical analysis of the fundamental frequencies. Appl. Stand. Mapp. Physica D 56, 253-269 (1992)

Lega, E., Guzzo, M., Froeschlé, C.: Detection of Arnold diffusion in Hamiltonian systems. Physica D 182, 179187 (2003)

Lega, E., Guzzo, M., Froeschlé, C.: Measure of the exponential splitting of the homoclinic tangle in fourdimensional symplectic mappings. Celest. Mech. Dyn. Astron. 104, 191-204 (2009)

Locatelli, U., Giorgilli, A.: Construction of Kolmogorov's normal form for a planetary system. Regul. Chaotic Dyn. 10, 153-171 (2005)

Locatelli, U., Giorgilli, A.: Invariant tori in the Sun-Jupiter-Saturn system. Discrete Contin. Dyn. Syst. Ser. B 7(2), 377-398 (2007)

Lochak, P.: Canonical perturbation theory via simultaneous approximation. Russ. Math. Surv. 47, 57-133 (1992)

Morbidelli, A., Giorgilli, A.: On the role of high order resonances in normal forms and in separatrix splitting. Physica D 102, 195-207 (1997) 
Nekhoroshev, N.N.: Exponential estimates of the stability time of near-integrable Hamiltonian systems. Russ. Math. Surv. 32, 1-65 (1977)

Neishtadt, A.I.: The separation of motions in systems with rapidly rotating phase. J. Appl. Math. Mech. 48, 133139 (1984)

Pavlovic, R., Guzzo, M.: Fulfilment of the conditions for the application of the Nekhoroshev theorem to the Koronis and Veritas asteroid families. Mon. Not. R. Astron. Soc. 384, 1575-1582 (2008)

Pöschel, J.: Nekhoroshev estimates for quasi-convex Hamiltonian systems. Math. Z. 213, 187 (1993)

Poincaré, H.: Les méthodes nouvelles de la Mécanique Céleste. Gauthier-Villars, Paris (1892)

Robutel, P.: Stability of the planetary three-body problem. II: KAM theory and the existence of quasiperiodic motions. Celest. Mech. Dyn. Astron. 62, 219-261 (1995)

Robutel, P., Gabern, F.: The resonant structure of Jupiter's Trojan asteroids I. Long-term stability and diffusion. MNRAS 372, 1463-1482 (2006)

Sidlichovský, M., Nesvorný, D.: Frequency modified Fourier transform and its applications to asteroids. Celest. Mech. Dyn. Astron. 65(1-2), 137-148 (1997)

Simó, C.: Averaging under fast quasi-periodic forcing. In: Seimenis, I. (ed.) Integrable and Chaotic Behaviour in Hamiltonian Systems, pp. 13-34, Plenum Pub. Co., New York (1994)

Simó, C.: Analytical and numerical detection of exponentially small phenomena. In: International Conference on Differential Equations, Vol. 1, 2 (Berlin, 1999), pp. 967-976. World Scientific Publications, River Edge, NJ (2000)

Simó, C., Valls, C.: A formal approximation of the splitting of separatrices in the classical Arnold's example of diffusion with two equal parameters. Nonlinearity 14, 1707-1760 (2001)

Todorovic̀, N., Lega, E., Froeschlé, C.: Local and global diffusion in the Arnold web of a priori unstable systems. Celest. Mech. Dyn. Astron. 102, 13-27 (2008) 